\title{
Angiotensin converting enzyme 2 (ACE2) is expressed in murine cutaneous under single-cell transcriptome resolution
}

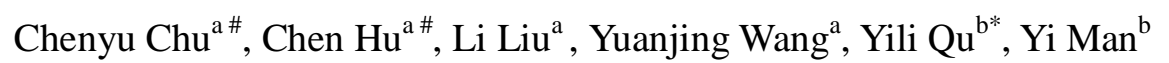 \\ ${ }^{a}$ Department of Oral Implantology \& National Clinical Research Center for Oral \\ Diseases \& State Key Laboratory of Oral Diseases, West China Hospital of \\ Stomatology, Sichuan University, Chengdu 610041, China. \\ \# These authors contributed equally to this work.
}

\section{Corresponding author:}

Yili Qu, DDS, PhD

Associate Professor

State Key Laboratory of Oral Diseases \& National Clinical Research Center for

Oral Diseases, West China Hospital of Stomatology, Sichuan University, 14\#, 3rd

section, Renmin South Road

Chengdu 610041, China

Tel: 0086-28-85503571

Email: qqyili@126.com

Yi Man, DDS, PhD

Chair and Professor

State Key Laboratory of Oral Diseases \& National Clinical Research Center for

Oral Diseases \& Department of Oral Implantology, West China Hospital of

Stomatology, Sichuan University

Tel: 0086-28-85503571

Email: Manyi780203@126.com 


\title{
Keywords: Angiotensin converting enzyme 2, mouse, skin
}

\author{
Abstract \\ Angiotensin converting enzyme 2 (Ace2) is widely distributed in human organs, \\ which was identified as a functional receptor for severe acute respiratory syndrome \\ (SARS) coronavirus in human beings. It was also confirmed that SARS-CoV-2 uses \\ the same cell entry receptor, ACE2, as SARS-CoV. However, related research still not \\ discover the expression data associated with murine skin under single cell RNA \\ resolution. In this study, we performed single-cell RNA sequencing (scRNA-seq) on \\ unsorted cells from mouse dorsal skin after 7 days post-wounding. 8312 sequenced \\ cells from four skin samples met quality control metrics and were analyzed.
}

\section{Introduction}

Angiotensin converting enzyme 2 (Ace2) is widely distributed in human organs, especially cardio-renal tissues, the digestive systems and respiratory tract. [1-4] It functions as a membrane-associated extracellular protein that cleaves angiotensin (Ang) I to generate Ang 1-9.[2] Later, Ace2 was identified as a functional receptor for severe acute respiratory syndrome (SARS) coronavirus [5, 6]. In mouse, SARS-Cov Spike protein bind to Ace2, resulting in aggravated acute lung failure through deregulation of the renin-angiotensin system. In the recent 2019 coronavirus epidemic (COVID-19) caused by a novel virus SARS-CoV-2, 2129 people had died by 20 February. Infections worldwide have topped 75000; more than 74000 are in China.[7] It was confirmed that SARS-CoV-2 uses the same cell entry receptor, ACE2, as

SARS-CoV.[8] To explore the expression of Ace2 in mouse skin wound model and 
potential functional roles of it, we performed single-cell RNA sequencing (scRNA-seq) on unsorted cells from mouse dorsal skin after 7 days post-wounding. 8312 sequenced cells from four skin samples met quality control metrics and were analyzed.

\section{Method and materials}

\subsection{Experimental model}

All animal procedures were approved by the Institutional Ethics Committee of Sichuan University. Animals included C57BL/6 male mice at ages from 6 to 8 weeks. Hair at the surgical area was removed by plucking against the growth direction. Full-thickness circular excisional wound (diameter=6mm) was created at the dorsal skin of mice. A sterile Tegaderm film (3M) was placed above the wound to protect the wound area. Then annular silicone splints (inner diameter $=8 \mathrm{~mm}$, outer diameter $=12 \mathrm{~mm}$ ) were sutured with the Tegaderm film and underlying skin in order to minimize the contraction of the dorsal muscle. After healing for a week, mice were euthanized for sample harvest.

\subsection{Specimen harvest}

We obtained skin samples by cutting off skin at the wound area (circular, diameter $=10 \mathrm{~mm}$ ). Subcutaneous tissues were removed. A total of four tissues were harvested. The tissues were washed in a $100 \mathrm{~mm}$ petri dish containing $20 \mathrm{ml}$ of phosphate-buffered saline (PBS). Then they were transferred to a $50 \mathrm{~mm}$ petri dish containing $100 \mu \mathrm{L}$ of Enzyme G (Epidermis Dissociation Kit mouse, Miltenyi) and 3.9 $\mathrm{ml}$ of PBS buffer with the dermal side facing downwards. Tissues were digested for 
16 hours at $4^{\circ} \mathrm{C}$. Then they were transferred into a $50 \mathrm{~mm}$ petri dish containing $4 \mathrm{~mL}$ of $1 \times$ Buffer S (Miltenyi). Epidermis was peeled off from the skin using tweezers, and was cut into pieces. Enzyme mix containing $3.9 \mathrm{ml}$ of $1 \times$ Buffer S, $100 \mu 1$ of Enzyme P, and $20 \mu$ of Enzyme A (Miltenyi) stored in a gentleMACS ${ }^{\mathrm{TM}} \mathrm{C}$ Tube was used to digest the epidermis pieces for 20 minutes at $37 \square$. Stop enzymatic reaction by adding $4 \mathrm{ml}$ of PBS that contain $0.5 \%$ bovine serum albumin (BSA). A gentleMACS dissociator (Miltenyi) was applied to automatically dissociate the epidermis (Program B). The sample was passed through a $70 \mu \mathrm{m}$ cell strainer (Corning), centrifuged at $300 \times \mathrm{g}$ for 10 minutes at room temperature, and suspended with PBS that contain $0.5 \%$ BSA. Cells were gently washed twice and stored in an ice box. For the dermis part, they were first cut into pieces (diameter $<1 \mathrm{~mm}$ ). The tissue was mixed with $10 \mathrm{ml}$ enzyme mix containing Type I Collagenase (3125U/Ml, Gibco) and 2.5ml trypsin (Gibco), and poured into a gentleMACS ${ }^{\mathrm{TM}} \mathrm{C}$ Tube. After dissociating the tissue on gentleMACS dissociator for 37s (Skin mode), another 10ml enzyme mix was added. The sample was digested for 2.5 hours at $37 \square$ in a rotary machine (Peqlab). Then the dermis sample was passed through a $70 \mu \mathrm{m}$ cell strainer (Corning), centrifuged at $300 \times \mathrm{g}$ for 5 minutes at room temperature, and suspended with $3 \mathrm{ml}$ red blood cell lysis buffer (Solarbio). After 3 minutes, the cell suspension was centrifuged and gently suspended with RPMI 1640 medium (Hyclone). Cells were gently washed twice with PBS containing $0.5 \%$ BSA and stored in an ice box. The epidermis and dermis cell solutions were mixed together as a whole. The sample was centrifuged, and suspended with $100 \mu 1$ Dead Cell Removal MicroBeads (Miltenyi). After incubation 
for $15 \mathrm{~min}$ at room temperature, the cell suspension was diluted in $3 \mathrm{ml} 1 \times$ Binding buffer (Miltenyi). LS columns (Miltenyi) were used for removal of dead cells and debris. The negatively selected live cells pass through the column, and were suspended with PBS containing $0.05 \%$ BSA. Finally, we proceeded with the 10x Genomics® Single Cell Protocol.

\subsection{Single-cell encapsulation and library generation}

Single cells were encapsulated in water-in-oil emulsion along with gel beads coated with unique molecular barcodes using the 10x Genomics Chromium Single-Cell Platform. For single-cell RNA library generation, the manufacturers' protocol was performed. (10×Single Cell 3' v3) Sequencing was performed using a NovaSeq PE150 mode with 94574 reads per cell. The Cell Ranger software was used to align reads and generate expression matrices for downstream analysis.

\section{Results}

Unsupervised clustering using Seurat categorized the cells into 21 clusters based on global gene expression patterns. Using the differentially expressed gene signatures, we annotated these clusters (Fig. 1a). 10\% cells from wounded skin express Ace2 (Fig.1b), the majority of which belong to keratinocytes (cluster 2, marker genes: Krt1, Krt10, Loricrin) in the epidermis (9) and sebocytes in fig. 2 (cluster 12, marker genes: Mgst1, Scd1, Dhcr24, Elovl6) (10). We also analyzed bulk RNA-Seq profiles of mouse healthy dorsal skin. ACE2 was expressed in healthy skin as well (Fragments per kilobase million (FPKM) values more than 1). According to Gene Ontology (GO) analysis, cluster 2 (keratinocyte) was enriched for genes related to keratinocyte 
differentiation, skin development, epidermis development, establishment of skin barrier, and keratinization (Fig. 3). The epidermis is a stratified epithelium composed of several layers of keratinocytes, which provides a physical barrier between the environment and the organism, thereby protecting it from external agents and pathogens, and limiting the loss of fluids.[9] Cornification is achieved by keratinocytes passing through 4 cell layers of the epidermis: the stratum basale, the stratum spinosum, the stratum granulosum (SG), and the stratum corneum in fig.4 (SC) [10]. Interestingly, Ace2+ keratinocytes (44\%) in cluster 2 is featured by up-regulated expression of keratins, e.g. Krt10, Krt78, which are markers for differentiated keratinocytes located at suprabasal layers of epidermis [9]. While Ace2- keratinocytes (56\%) are featured by high expression level of Late cornified envelope group I (LCE1) genes, filaggrin2 (Flg2) and Loricrin (Fig. 5), the encoded protein of which are key elements for cornified cell envelope (CE). As SG keratinocytes transition into SC, the keratinocytes become flattened and denucleated (which are then called corneocytes). Lamellar bodies (containing extracellular components, e.g. lipids, corneodesmosin, and kallikreins) are secreted into the intercellular space between the corneocytes and fill with lipid. These structures are often described as bricks (corneocytes) and mortar (intercellular lipids), which together provide a highly hydrophobic barrier, namely cornified cell envelope (CE), against the environment [10-12]. The gene expression profiles of Ace2+ and Ace2- cells indicate that keratinocytes in the spinous and granular layers of epidermis express Ace2, while corneocytes at the outermost cornified layer do not. GO analysis of cluster 12 (sebocytes) exhibited enriched gene 
expression related to cellular respiration, ATP metabolic process and oxidative phosphorylation (Fig. 3). Cellular respiration, through oxidative phosphorylation (OXPHOS), constitutes the main oxygen-consuming and ATP-producing processes [13] .

\section{Discussion}

Gene enrichment in these cellular metabolic processes indicates active energy-consuming processes in sebaceous gland after skin wounding. This might be related to function of sebocytes. Mouse sebocytes produce sebum (containing triglycerides, diglycerides, and free fatty acids, wax esters, and cholesterol) in a holocrine approach, which is crucial for hair growth, moisturization of skin and hair, and the prevention of water evaporation from the skin surface $[14,15]$. The sebaceous gland arises as an outgrowth of the outer root sheath of the hair follicle as undifferentiated sebocytes emerge from peripheral cells and then move centrally (central cells) as first partially, and later fully, differentiated sebocytes in fig.4 [16]. The cuboidal or flattened peripheral cells contain no lipids, while the matured central cells have a frothy appearance as a result of the accumulation of cytoplasmic lipids.[14] In cluster 12 (sebocyte), Ace2+ cells (26\%) showed up-regulated genes including stearoyl-CoA desaturase 1 (Scd1), Scd3, cell death-inducing DNA fragmentation factor (DFFA)-like effector (CIDE) a (Cidea), elongation of very-long-chain fatty acid protein 3 (Elovl3), and phosphatidylcholine transfer protein (Pctp), in comparison with Ace ${ }^{+}$cells in fig.6 (74\%). Among them, Scd1 and Scd3 are specifically expressed in the sebaceous gland of mouse skin [17, 18]. Previous 
studies demonstrated that Scd1 expressing sebocytes derive from precursor cells mobilised from the hair follicle bulge. The precursor cells are marked by expression of Lrig1 [19]. When we overlap cells that respectively express Lrig1, Scd1 and Scd3 with Ace $2^{+-}$cells in Umap, we found that the Lrig1 is more sufficiently expressed in Ace2 ${ }^{-}$cells (Fig. ?), suggesting that these cells might be located at peripheral zones in the sebaceous gland. Among the up-regulated genes in Ace $2^{+}$cells, protein of Elovl3 and Scd1 regulate lipid synthesis. Cidea is a crucial regulator of sebaceous gland lipid storage and sebum lipid secretion [20]. These functional genes are up-regulated in sebocytes expressing Ace2, which further indicate that they are matured central cells in sebaceous gland. We also observed that sebocytes resemble adipocytes in gene expressions despite their differences in lipid production, composition, and release. The afore-mentioned Elovl3 and Cidea were also marker genes for brown adipocytes. Pctp is up-regulated in $\mathrm{Ace} 2^{+}$cells, and has a critical role in regulating mitochondrial oxidation of fatty acids in metabolically active tissues, e.g. it is crucial for regulating adaptive thermogenesis in brown adipose tissue (BAT) [21-23]. Ace2- cells were featured by increased expression of mitochondrial 3-hydroxy-3-methylglutaryl CoA synthase 2 (Hmgcs2) and insulin-like growth factor (IGF) binding-protein 2 (Igfbp2). Hmgcs2 has been implicated in the synthesis of ketone bodies, which can regulate adipocyte browning [24]. It also contributes to cellular hydroxymethylglutaryl-coenzyme A (HMG-CoA) levels and thereby provides substrate for mevalonate synthesis, the inhibition of which affects uncoupling in mature human and murine brown adipocytes, mitochondrial respiration, and browning 
of the white adipocytes [25].The Igfbp2 protein is the predominant Igfbp produced by white preadipocytes during adipogenesis [26]. The level of Igfbp2 expressed by adipocytes was associated with fat mass, while circulating Igfbp2 was positively associated with insulin sensitivity [27, 28]. When assessing the effect of an epidemic, transmissibility and severity are the 2 most critical factors [29]. An estimated basic reproductive number (R0, a commonly used measure of transmissibility and is defined as the number of additional persons one case infects over the course of their illness) is 2.2, which indicates the infection has the potential for sustained transmission [30]. However, the transmission routes are still not fully clarified. As a target for SARS-CoV-2, the distribution of Ace2 might offer clues for exploring possible transmission routes. In the mouse wounded skin sample, cells that express Ace2 are suprabasal keratinocytes in the epidermis and sebum-secreting sebocytes in sebaceous glands. Whether the pathogen can infect the organism through skin contact, especially when the skin is wounded, remains to be verified.

\section{Funding information}

This work was supported by Research and Develop Program, West China Hospital of Stomatology Sichuan University (No.LCYJ2019-19); the Fundamental Research

Funds for the Central Universities (No. 2082604401239)

\section{Statement of conflict of interest}

There are no conflicts of interest related to this manuscript. 


\section{References:}

[1] Donoghue M. Heart block, ventricular tachycardia, and sudden death in ACE2 transgenic mice with downregulated connexins. Journal of Molecular and Cellular Cardiology. 2003;35:1043-53.

[2] Donoghue M, Hsieh F, Baronas E, Godbout K, Gosselin M, Stagliano N, et al. A novel angiotensin-converting enzyme-related carboxypeptidase (ACE2) converts angiotensin I to angiotensin 1-9. Circulation research. 2000;87:E1-9.

[3] Zhang H, Kang Z, Gong H, Xu D, Wang J, Li Z, et al. 2020.

[4] Hamming I, Timens W, Bulthuis ML, Lely AT, Navis G, van Goor H. Tissue distribution of ACE2 protein, the functional receptor for SARS coronavirus. A first step in understanding SARS pathogenesis. The Journal of pathology. 2004;203:631-7. [5] Li W, Moore MJ, Vasilieva N, Sui J, Wong SK, Berne MA, et al. Angiotensin-converting enzyme 2 is a functional receptor for the SARS coronavirus. Nature. 2003;426:450-4.

[6] Kuba K, Imai Y, Rao S, Gao H, Guo F, Guan B, et al. A crucial role of angiotensin converting enzyme 2 (ACE2) in SARS coronavirus-induced lung injury. Nature medicine. 2005;11:875-9.

[7] <20200220-sitrep-31-covid-19.pdf>.

[8] Zhou P, Yang X-L, Wang X-G, Hu B, Zhang L, Zhang W, et al. 2020.

[9] Pastar I, Stojadinovic O, Yin NC, Ramirez H, Nusbaum AG, Sawaya A, et al. Epithelialization in Wound Healing: A Comprehensive Review. Advances in wound care. $2014 ; 3: 445-64$. 
[10] Egawa G, Kabashima K. Multifactorial skin barrier deficiency and atopic dermatitis: Essential topics to prevent the atopic march. The Journal of allergy and clinical immunology. 2016;138:350-8 e1.

[11] Ishitsuka Y, Huebner AJ, Rice RH, Koch PJ, Speransky VV, Steven AC, et al. Lce1 Family Members Are Nrf2-Target Genes that Are Induced to Compensate for the Loss of Loricrin. The Journal of investigative dermatology. 2016;136:1656-63.

[12] Deng Z, Matsuda K, Tanikawa C, Lin J, Furukawa Y, Hamamoto R, et al. Late Cornified Envelope Group I, a novel target of p53, regulates PRMT5 activity. Neoplasia. 2014;16:656-64.

[13] McClelland GB. TISSUE RESPIRATION | Cellular Respiration. 2011:951-8.

[14] Smith KR, Thiboutot DM. Thematic review series: skin lipids. Sebaceous gland lipids: friend or foe? Journal of lipid research. 2008;49:271-81.

[15] Schneider MR, Paus R. Sebocytes, multifaceted epithelial cells: lipid production and holocrine secretion. The international journal of biochemistry \& cell biology. 2010;42:181-5.

[16] Niemann C. Differentiation of the sebaceous gland. Dermato-endocrinology. 2009;1:64-7.

[17] <1999,Nature Genetics, Scd1 is expressed in sebaceous glands and is disrupted in the asebia mouse.pdf $>$.

[18] Zheng Y, Prouty SM, Harmon A, Sundberg JP, Stenn KS, Parimoo S. Scd3--a novel gene of the stearoyl-CoA desaturase family with restricted expression in skin. Genomics. 2001;71:182-91. 
[19] Frances D, Niemann C. Stem cell dynamics in sebaceous gland morphogenesis in mouse skin. Developmental Biology. 2012;363:138-46.

[20] Zhang S, Shui G, Wang G, Wang C, Sun S, Zouboulis CC, et al. Cidea control of lipid storage and secretion in mouse and human sebaceous glands. Molecular and cellular biology. 2014;34:1827-38.

[21] Kang HW, Ribich S, Kim BW, Hagen SJ, Bianco AC, Cohen DE. Mice lacking Pctp /StarD2 exhibit increased adaptive thermogenesis and enlarged mitochondria in brown adipose tissue. Journal of lipid research. 2009;50:2212-21.

[22] Scapa EF, Pocai A, Wu MK, Gutierrez-Juarez R, Glenz L, Kanno K, et al. Regulation of energy substrate utilization and hepatic insulin sensitivity by phosphatidylcholine transfer protein/StarD2. FASEB journal : official publication of the Federation of American Societies for Experimental Biology. 2008;22:2579-90.

[23] Kang HW, Wei J, Cohen DE. PC-TP/StARD2: Of membranes and metabolism. Trends in endocrinology and metabolism: TEM. 2010;21:449-56.

[24] Carriere A, Jeanson Y, Berger-Muller S, Andre M, Chenouard V, Arnaud E, et al. Browning of white adipose cells by intermediate metabolites: an adaptive mechanism to alleviate redox pressure. Diabetes. 2014;63:3253-65.

[25] Balaz M, Becker AS, Balazova L, Straub L, Muller J, Gashi G, et al. Inhibition of Mevalonate Pathway Prevents Adipocyte Browning in Mice and Men by Affecting Protein Prenylation. Cell metabolism. 2019;29:901-16 e8.

[26] Li Z, Picard F. Modulation of IGFBP2 mRNA expression in white adipose tissue upon aging and obesity. Hormone and metabolic research $=$ Hormon- und 
Stoffwechselforschung = Hormones et metabolisme. 2010;42:787-91.

[27] Yau SW, Russo VC, Clarke IJ, Dunshea FR, Werther GA, Sabin MA. IGFBP-2

inhibits adipogenesis and lipogenesis in human visceral, but not subcutaneous, adipocytes. International journal of obesity. 2015;39:770-81.

[28] Claudio M, Benjamim F, Riccardo B, Massimiliano C, Francesco B, Luciano C. Adipocytes IGFBP-2 expression in prepubertal obese children. Obesity. 2010;18:2055-7.

[29] Swerdlow DL, Finelli L. Preparation for Possible Sustained Transmission of 2019 Novel Coronavirus: Lessons From Previous Epidemics. Jama. 2020.

[30] Li Q, Guan X, Wu P, Wang X, Zhou L, Tong Y, et al. Early Transmission Dynamics in Wuhan, China, of Novel Coronavirus-Infected Pneumonia. The New England journal of medicine. 2020. 
Figure legends

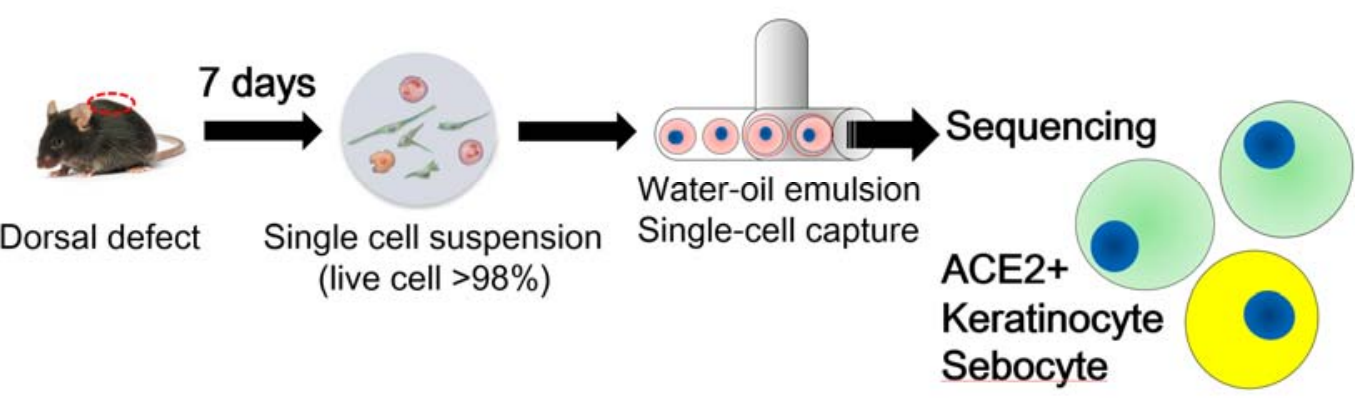

Illustration of whole experimental design and cell-clustering process under single cell RNA-seq level 


\section{Illustration of experiments}
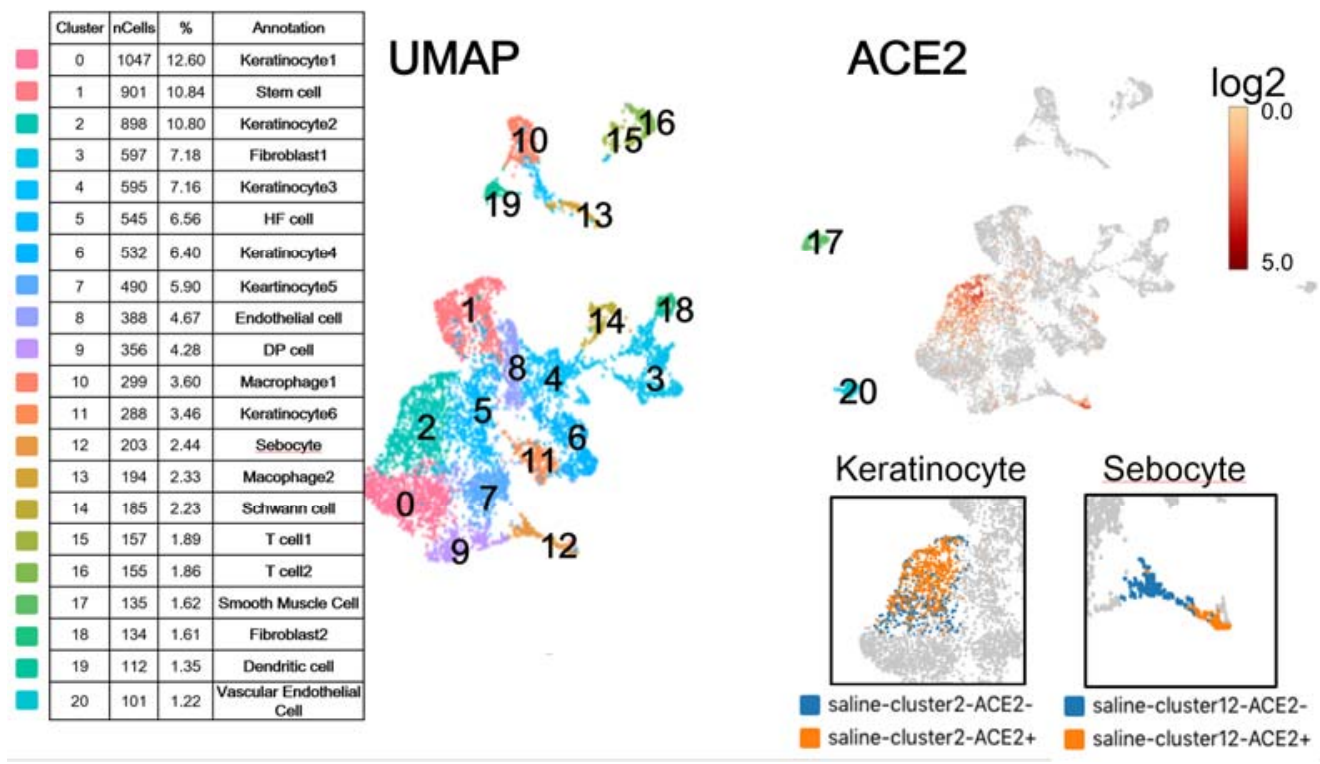

Fig. 1 Clustering ACE2-positive area in UMAP, further marked related cells as keratinocyte and sebocyte, which express highly ACE2 level in murine

full-thickness skin wound healing process at day 7 

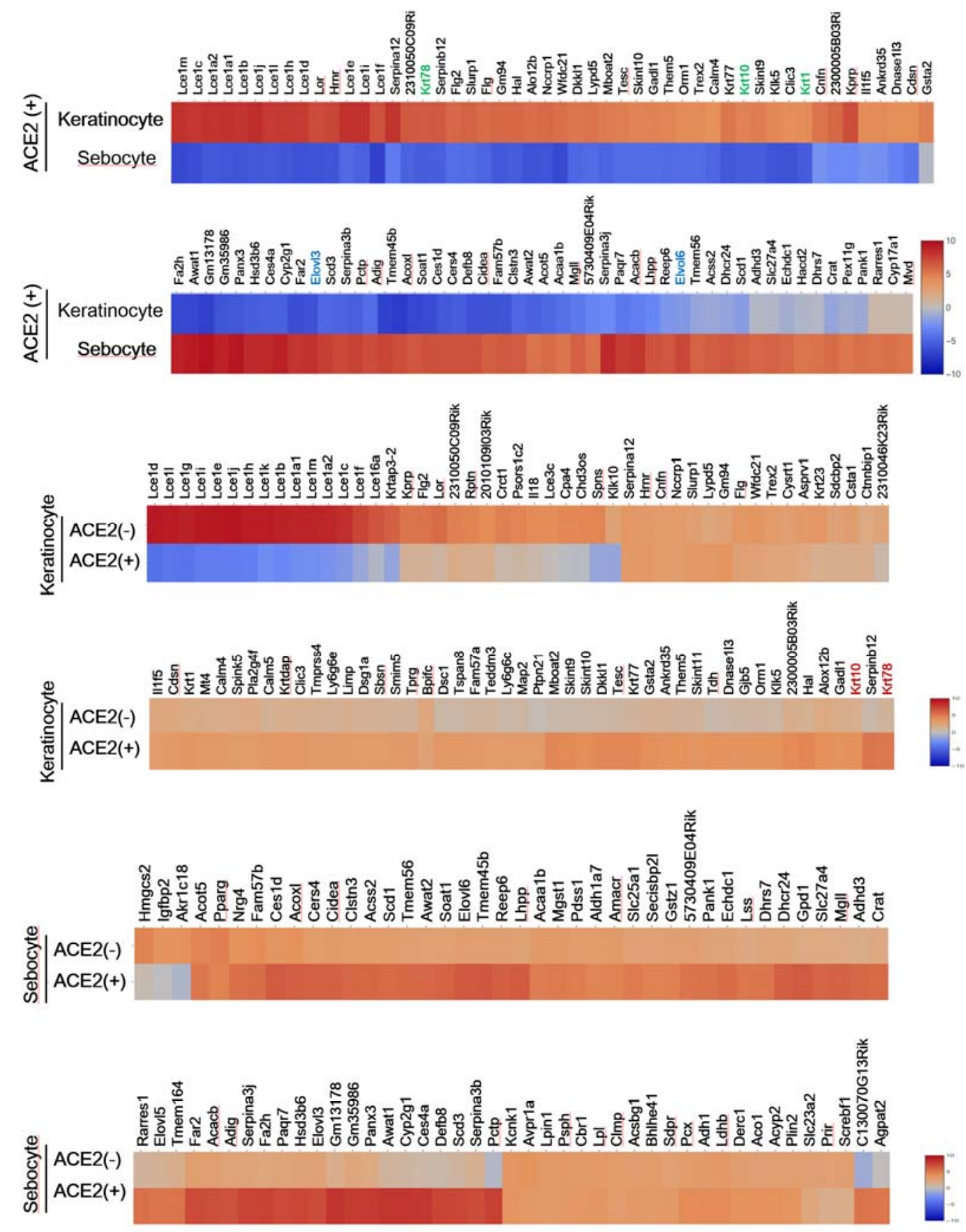

Figure 2 Highly different gene expression in both keratinocyte and sebocyte, further analysis the ACE2 positive and negative subset, spatial specificity genes, including krt10, krt78, krt1 etc, further implication of ACE2 expression related to location in full-thickness and degree of maturation in murine skin healing process. 
bioRxiv preprint doi: https://doi.org/10.1101/2021.10.07.463533; this version posted October 8, 2021. The copyright holder for this preprint (which was not certified by peer review) is the author/funder, who has granted bioRxiv a license to display the preprint in perpetuity. It is made available under aCC-BY-NC-ND 4.0 International license.
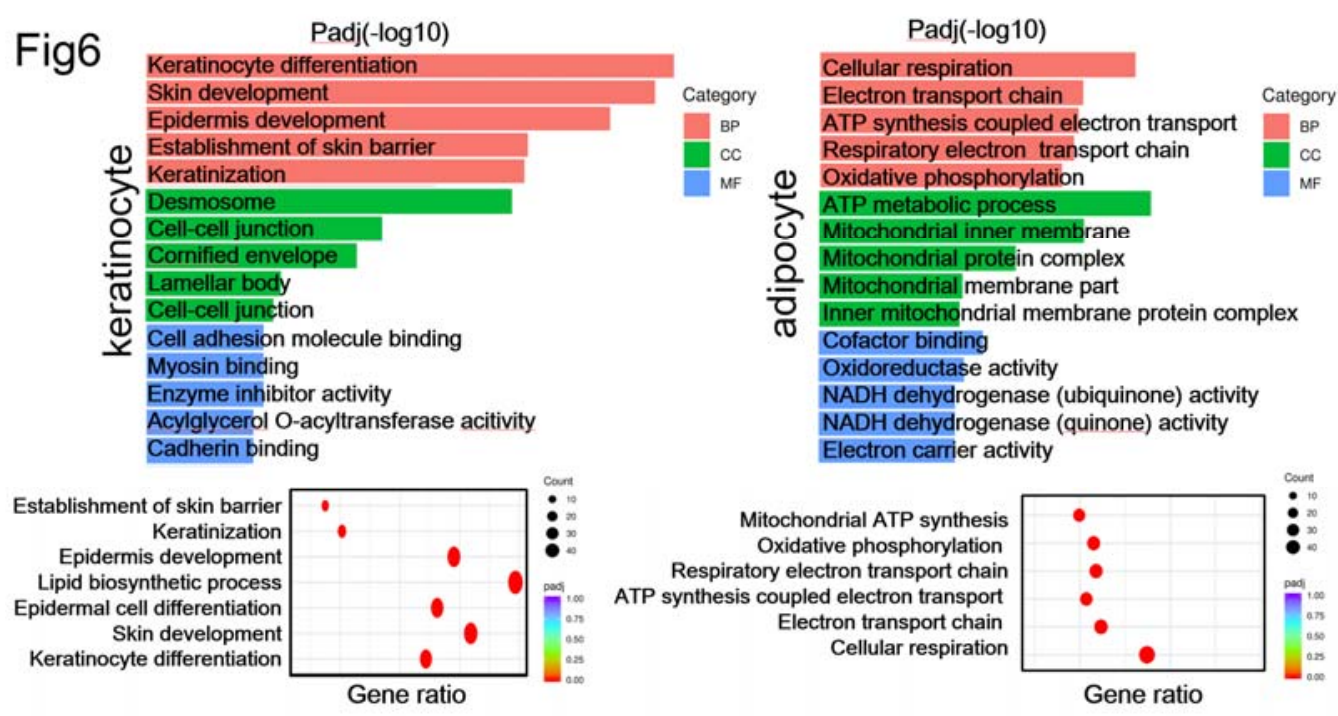

Figure 3 KEGG and GO analysis implication of the function and signaling pathway in

different cell types highly expressed ACE2 


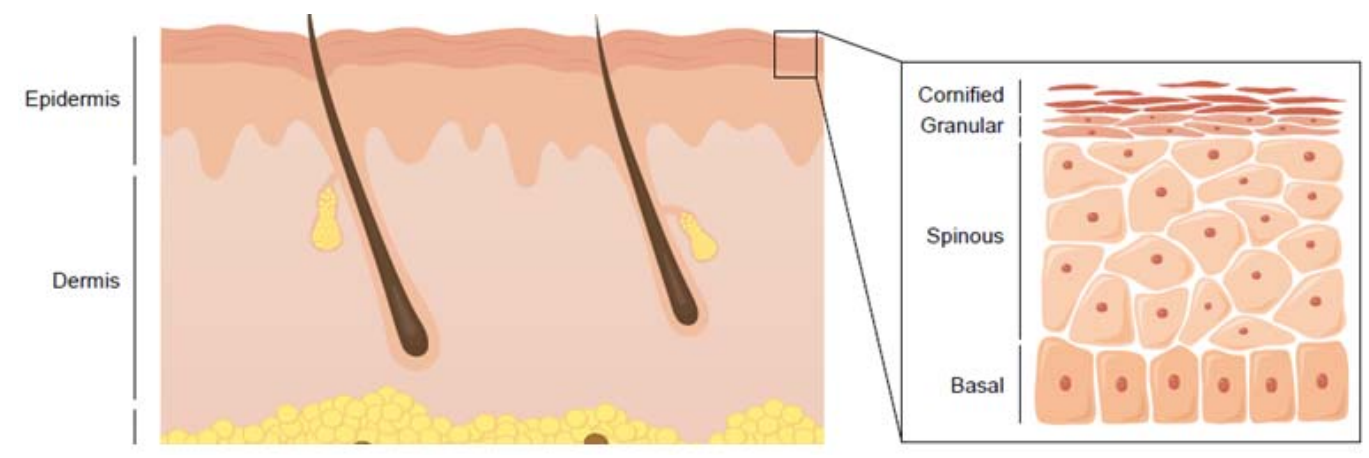

Figure 4 Spatial specific gene expression in different layer of murine skin, krt1 and krt5

which presented the early stage of keratinocyte maturation, i.e., which is low-differentiation

keratinocyte, highly expressed in basal layer. However, the classic late-stage differentiation

marker of keratinocyte, krt10 or newly reported specific marker krt78, expressed in outer

layer, including granular and comified layer. 


\section{A. Keratinocyte}
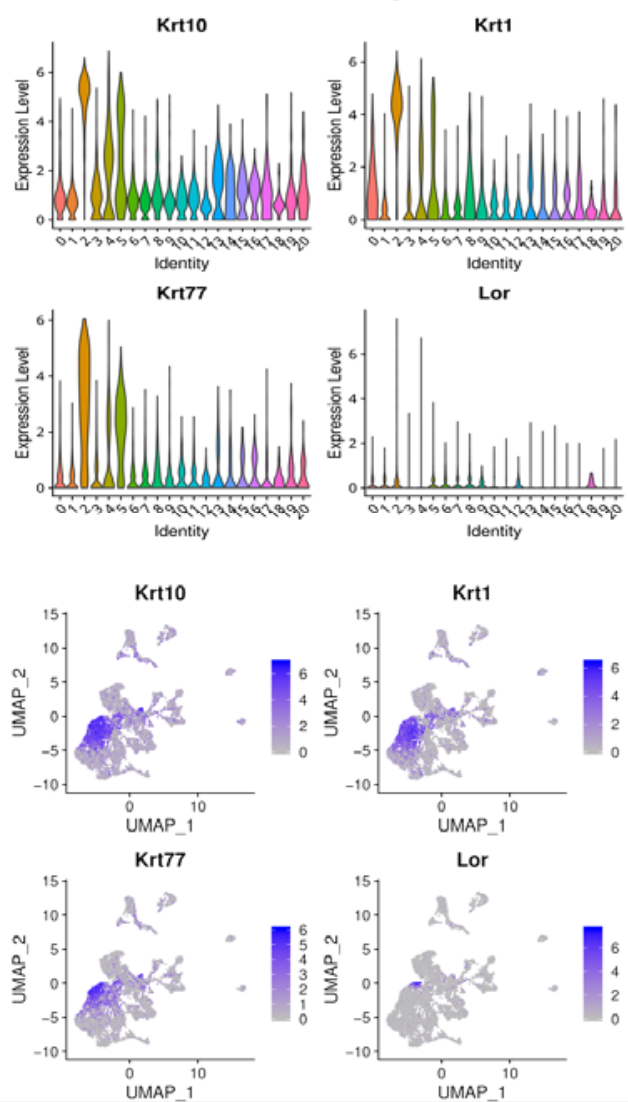

\section{B. Sebocyte}
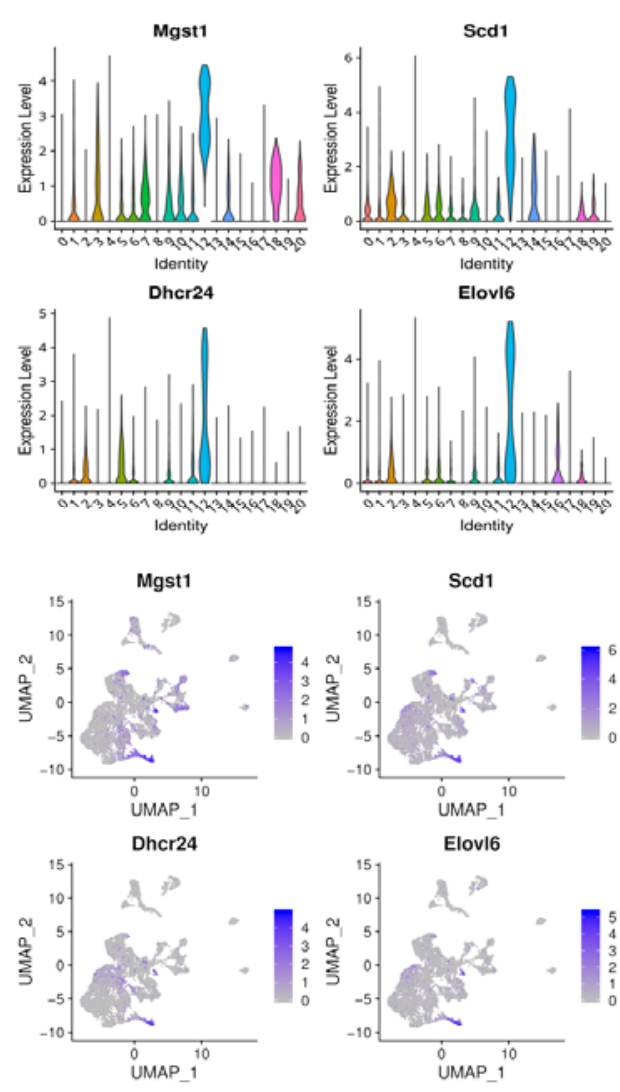

Figure 5. Top 4 high expression gene in different cell type, and the expression area in UMAP. 